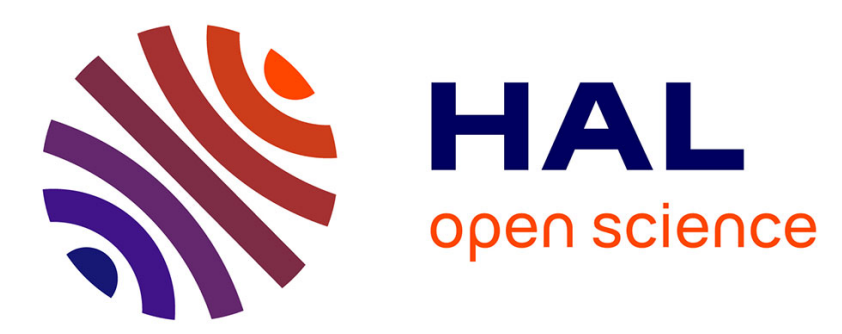

\title{
Quel potentiel des personnes face à la grande mobilité ? Réflexions exploratoires autour de la notion de motilité
}

\author{
Yann Dubois, Emmanuel Ravalet, Vincent Kaufmann
}

\section{To cite this version:}

Yann Dubois, Emmanuel Ravalet, Vincent Kaufmann. Quel potentiel des personnes face à la grande mobilité ? Réflexions exploratoires autour de la notion de motilité. RTS - Recherche Transports Sécurité, 2015, Usages de la vitesse, 2015 (01), pp.47-61. 10.4074/S0761898015001053 hal-01670603

\section{HAL Id: hal-01670603 https://hal.science/hal-01670603}

Submitted on 21 Dec 2017

HAL is a multi-disciplinary open access archive for the deposit and dissemination of scientific research documents, whether they are published or not. The documents may come from teaching and research institutions in France or abroad, or from public or private research centers.
L'archive ouverte pluridisciplinaire HAL, est destinée au dépôt et à la diffusion de documents scientifiques de niveau recherche, publiés ou non, émanant des établissements d'enseignement et de recherche français ou étrangers, des laboratoires publics ou privés. 


\title{
Quel potentiel des personnes face à la grande mobilité ? Réflexions exploratoires autour de la notion de motilité
}

\section{What potential do people have for high mobility? An exploratory study on the concept of motility}

\author{
Yann Dubois · Emmanuel Ravalet · Vincent Kaufmann
}

(C) IFSTTAR et Éditions NecPlus 2015

Résumé Le développement des offres en matière de transports et de technologies de la communication permettent aux personnes de se déplacer toujours plus, plus loin et plus rapidement. La flexibilisation du marché du travail impose l'utilisation de ces potentiels et favorise la mobilité. Plus spécifiquement, elle accompagne l'émergence de pratiques de grande mobilité, telles que la pendularité de longue durée, la bi-résidentialité ou encore les voyages fréquents pour motif professionnel. Dans ce contexte, les personnes ne sont pas toutes égales face à la grande mobilité. Basé sur l'enquête quantitative longitudinale Job Mobilities and Family Lives réalisée en 2007 puis en 2011, cet article, dans une perspective transversale et longitudinale, vise à explorer le potentiel de (grande) mobilité des personnes, à l'aide du concept de «motilité ». Premièrement, les résultats montrent que la motilité permet de décrire finement les situations des personnes dans la perspective d'un recours éventuel à la grande mobilité. Alors que certains sont dans une position confortable face à la grande mobilité, d'autres en revanche combinent de mauvaises compétences de mobilité et des accès limités. Même s'ils se déclarent disposés à la grande mobilité, ils apparaissent comme plus vulnérables. Deuxièmement, l'évolution du potentiel de mobilité est avant tout portée par des changements dans les projets de mobilité : les contextes familiaux, économiques et spatiaux dans lesquels les personnes se trouvent expliquent ces

\section{Yann Dubois $(\triangle)$}

Laboratoire de Sociologie Urbaine, EPFL

Bâtiment BP, Station 16, CH-1015-Lausanne, Suisse

E-mail : yann.dubois@epfl.ch

Emmanuel Ravalet $(\square)$

Laboratoire de Sociologie Urbaine, EPFL

Bâtiment BP, Station 16, CH-1015-Lausanne, Suisse

E-mail : emmanuel.ravalet@epfl.ch

Vincent Kaufmann $(\triangle)$

Laboratoire de Sociologie Urbaine, EPFL

Bâtiment BP, Station 16, CH-1015-Lausanne, Suisse

vincent.kaufmann@epfl.ch changements. La situation des Espagnols, particulièrement touchés par la crise dès 2008 , est à ce sujet très parlante. Finalement, l'analyse du lien entre motilité et mobilité montre, de manière contre-intuitive, qu'un plus grand potentiel de mobilité ne se traduit pas nécessairement par un recours plus important à la grande mobilité.

Mots-clés grande mobilité · pendularité · motilité · potentiel $\cdot$ accès $\cdot$ compétences $\cdot$ projets $\cdot$ contextes spatiaux $\cdot$ crise économique

Abstract Recent developments in transport systems and information technology applications enable people to move more frequently, faster, and further afield. The increasing flexibility of the labour market requires these new potentials to be pressed into use, with increased mobility as a result. Specific high mobility practices are emerging, such as daily long-duration commuting, weekly commuting and work-related frequent trips. However, people are not equal regarding high mobility. Based on the quantitative study Job Mobilities and Family Lives conducted in 2007 and 2011, this article combines longitudinal and cross-sectional perspectives to explore the potential of individuals for (high) mobility, using the concept of "motility". Results show that motility is able to explain to a significant extent the various situations regarding a possible resort to high mobility. Although some of the participants are in a good position to cope with high mobility, others combine poor access to mobility and poor mobility skills. Even if they declare being ready to move, they appear to be a vulnerable position. Further results show that the evolution of mobility potential is explained by changes in mobility projects: family, economic and spatial contexts are central to a better understanding of these changes. In this regard, the situation of the Spaniards, particularly affected from 2008 by the financial crisis, is striking. Finally, analysis of the link between motility and mobility underlines that a higher mobility potential often does not imply more frequent resort to high mobility. 
Keywords High mobility · Commuting · Motility · Potential · Access · Skills · Projects · Spatial context . Economic crisis

\section{Introduction}

En Occident depuis une vingtaine d'années, les personnes se déplacent plus vite, plus souvent et plus loin. Au-delà de la tendance générale, cette augmentation est en bonne partie due à une frange très mobile de la population souvent identifiée dans la littérature internationale par le vocable de « grands mobiles » $[1,2]$. Concrètement, dans le présent article, nous nous focaliserons sur les mobilités réversibles professionnelles que sont la pendularité de longue durée, la bi-résidentialité (aller-retour, une à plusieurs fois par semaine) et les voyages professionnels fréquents.

Ces déplacements sont en forte croissance. En Allemagne par exemple, la part des pendulaires de longue distance (trajet aller de plus de $50 \mathrm{~km}$ ) parmi les employés est ainsi passée de 2,6 \% à 6,6 \% entre 1985 et 1997 [3]. En Suisse, selon le relevé structurel de 2010 de l'Office Fédéral de la Statistique, près de $10 \%$ de la population active se déplace pendant plus de 2 heures par jour ; $10 \%$ font plus de 100 kilomètres par jour de déplacements domicile - travail. À l'échelle européenne, $7 \%$ des actifs réalisent des trajets pendulaires quotidiens de longue durée, c'est-à-dire supérieurs à deux heures de trajet aller-retour [4].

Les deux dernières décennies ont connu de profonds changements en matière de mobilité. Trois faits congruents expliquent cette situation :

1. l'accroissement des échanges internationaux. La mondialisation a contribué à l'augmentation des flux économiques (financiers, mais également de marchandises) et à un accroissement de l'interdépendance entre les économies, favorisé par les accords de libre-échange internationaux ;

2. l'amélioration des transports et des systèmes de communication a permis d'intensifier ces échanges. Les systèmes de transports, qu'ils soient routiers, aériens ou ferroviaires se sont considérablement développés, permettant des gains notables en matière de coûts, de capacités et surtout de vitesse. L'apparition des compagnies aériennes low cost et la croissance forte des déplacements qu'elles rendent possibles, symbolisent parfaitement cet accès facilité et démocratisé à la vitesse. Les potentiels de mobilité qu'ont offerts et qu'offrent ces améliorations permettent de combiner et de connecter ce qui autrefois était socialement et spatialement inconciliable [5]. Ces éléments ont participé à une accélération générale des modes de vie contemporains [6,7], mais ont aussi profondément transformé les déplacements des personnes, en termes de fréquence, de portée spatiale et de vitesses ;

3. une transformation du monde du travail, avec d'une part une croissance de la précarité, en particulier l'accroissement des contrats à durée déterminée. D'autre part, une exigence accrue de flexibilité dans les horaires et un accroissement du niveau de chômage. Cette précarisation du marché du travail a institué la mobilité et la flexibilité comme des valeurs sociales fondamentales, comme des normes sociales [8]. Ainsi, un employeur attend que ses employés acceptent de se déplacer dans le cadre de leur travail, qu'il s'agisse de courts déplacements ou de séjours plus longs à l'étranger [9]. De même, dans la plupart des sociétés européennes, les personnes en situation de recherche d'emploi sont tenues d'être prêtes à se déplacer pour retrouver du travail. Par ailleurs, la migration est vivement encouragée par la Commission Européenne à l'échelle continentale pour optimiser le fonctionnement des différents marchés nationaux du travail et de l'économie européenne en général [10].

Ces trois faits congruents constituent à bien des égards une injonction à la mobilité, injonction qui pousse les salariés à se déplacer davantage. Le temps moyen passé à se déplacer dans la vie quotidienne a augmenté en Europe ces dernières années [11-14]. Ainsi, il apparaît que les gains de vitesse sont souvent réaffectés dans la distance ou des programmes d'activités toujours plus complexes [15], et en particulier par les pratiques de grande mobilité. Le recours croissant à la vitesse, à travers la grande mobilité dans un contexte économique difficile et exigeant de la flexibilité, questionne sur la manière dont les individus peuvent - et sont préparés à - y faire face. L'explorer est l'objet du présent article.

Nous abordons cette question à partir des données de panel de la recherche Job Mobilities and Family Lives (ci-après abrégée en JobMob), menée dans 4 pays européens (Allemagne, France, Espagne et Suisse) en 2007 et 2011. Après une première partie consacrée à la revue de la littérature, nous discuterons des modalités et difficultés liées à la mesure de la motilité. La partie « résultats » est découpée en quatre temps. Le premier temps porte sur l'analyse du potentiel des individus à être grand mobile, à travers la notion de motilité, et met en évidence plusieurs profils de motilité. Le deuxième se penche plus précisément sur les liens entre motilité et localisation résidentielle. Nous considérons ensuite l'évolution dans le temps de la motilité. Finalement, c'est le lien entre la motilité et la grande mobilité qui est examiné. 


\section{Problématique : entre localisations et motilité}

Pour étudier la mobilité et la grande mobilité, la prise en compte des lieux dans lesquels les pratiques quotidiennes des individus prennent place, et notamment les lieux de résidence et d'emploi, est indispensable.

La littérature sur la mobilité et les localisations résidentielles, au-delà des approches visant à analyser et quantifier les flux entre villes, régions ou quartiers, s'est également penchée sur les questions des choix résidentiels et les logiques qui les portent. Ainsi, le choix résidentiel et plus fondamentalement le fait de déménager apparaissent en premier lieu comme des décisions importantes et souvent difficiles à prendre dans l'histoire d'un individu ou d'un couple [16]. Le déménagement et la migration étant des formes de mobilité qui sont particulièrement impliquantes socialement et spatialement parlant, elles ne peuvent se répéter fréquemment, d'autant plus pour les personnes qui sont propriétaires de leur logement [17]. De même, les multiples ancrages et les connaissances spécifiques qui se construisent autour du logement ne favorisent pas le déménagement $[18,19]$.

La décision de déménager et le choix d'une localisation résidentielle se font, en général, en cherchant à prendre en compte les intérêts et les besoins des différents membres du ménage. Ainsi, le choix résidentiel sera influencé par les préférences propres à chacun des membres du ménage, leur(s) lieu(x) d'emploi(s) (actuels ou futurs), les localisations scolaires des enfants, etc. La littérature met en avant des facteurs, tels que la localisation, les caractéristiques du logement, ou l'environnement social et physique du lieu de vie, pour expliquer les choix résidentiels ; l'importance accordée à chaque facteur variant selon les individus et les ménages [20].

La localisation du logement à proximité d'un panel de services, tels que commerces, banques, établissements scolaires, etc., est également un critère important des ménages pour le choix de leur logement, tout comme l'accessibilité au lieu de travail. Cependant, les zones les plus centrales et les mieux pourvues se révèlent parallèlement être bien souvent les plus chères pour le logement. Ainsi, certains quartiers, communes ou régions connaissent des prix de l'immobilier élevés, à la location ou à l'achat. Les ménages, selon les budgets à disposition pour le poste «logement », se voient de ce fait limités dans leur choix de localisation résidentielle. Ces tendances concourent ainsi à créer de la ségrégation résidentielle. D'un point de vue plus général, le marché de l'immobilier se caractérise par une adéquation difficile entre demande et offre de logement [21]. La structure du ménage va également être associée à des choix spécifiques en termes de caractéristiques du logement (taille, type, etc.), avec une propension plus importante de la part des familles pour se loger dans des maisons individuelles, célibataires ou couples sans enfant préférant généralement des logements plus petits ou des appartements, ce qui tend à générer une spécialisation «familiale » des espaces résidentiels [22]. Cette préférence pour la maison individuelle ou un logement plus spacieux, qui ne concerne d'ailleurs pas uniquement les familles, a participé au fil du temps à un étalement des espaces résidentiels des villes-centres vers les campagnes [23]. L'environnement physique et social $\mathrm{du}$ logement influence également le choix résidentiel. Les approches par les modes de vie, et notamment la littérature sur la gentrification, confèrent une grande importance au contexte résidentiel et à son adéquation avec les valeurs et les préférences des individus [24-26]. Le choix résidentiel, et les poids accordés aux différents éléments (localisation, caractéristiques du logement, environnement), sont intimement liés au parcours de vie : aux différentes étapes importantes dans la vie des individus et des ménages (départ du domicile familial, formation d'un couple, arrivée d'un enfant, etc.) correspondent différentes propensions à la mobilité résidentielle, différents besoins et différentes préférences en termes de localisation, de caractéristiques du logement et de son environnement immédiat [27].

Le lieu de travail est également déterminant dans la spatialisation des activités et des mobilités des personnes. Pour autant, le choix d'un emploi n'est que faiblement influencé par sa localisation. L'inverse vaut également : les ménages considèrent le lieu de travail souvent comme un facteur secondaire dans le choix de localisation résidentielle [17]. Ce constat se renforce dans un contexte où le marché de l'emploi est tendu : nombre limité d'offres adaptées au profil du demandeur d'emploi et augmentation des contrats plus courts et plus précaires (CDD, intérims, travail à temps partiel) [28].

Les couples de localisations logement/emploi, propres à chaque personne, évoluent dans le temps. Il n'est pas toujours aisé ou judicieux d'adapter sa localisation résidentielle à son lieu de travail, surtout dans un contexte où l'emploi est peu stable et temporaire. Par ailleurs, face au chômage ou à un contexte économique difficile, les personnes sont contraintes de recourir à des formes de grande mobilité, réversibles ou irréversibles [29, 30]. Ainsi, plus de la moitié des 20-59 ans dans une étude allemande ont déclaré avoir été contraints ou partiellement contraints de devenir mobiles [31]. Parallèlement, le recours à la migration ou à la pendularité sur de longues distances apparaît comme plus fréquent lorsque les personnes ont été en situation de chômage [32].

Dans un contexte où le marché de l'emploi et le marché du logement se tendent, les mobilités professionnelles s'amplifient (en fréquence et en distance) et les 
mobilités résidentielles se complexifient... Permise par l'augmentation de la vitesse des systèmes de transports et de communication, l'apparition des grandes mobilités est notamment due à une substitution croissante de la migration par des formes de pendularités de longue durée/distance et de bi-résidentialité [33, 34]. L'augmentation de la part de couples bi-actifs, qui ne travaillent pas nécessairement dans la même agglomération, celle des couples ne faisant pas ménage commun (living apart together) ou celle des familles recomposées participent au développement de ces nouvelles formes de mobilités [35-37]. Finalement, le recours à la grande mobilité s'explique aussi par l'expansion de la suburbanisation aux couronnes les plus éloignées des centres urbains et les moins connectées, où les terrains restent disponibles à des prix acceptables [34].

N'entrant pas dans les catégories traditionnelles d'appréhension des mobilités spatiales, que sont la mobilité quotidienne, les voyages, les mobilités résidentielles et les migrations [38], ces mobilités particulières sont relativement peu étudiées. En effet, la plupart des enquêtes statistiques sont peu adaptées pour les analyser en raison de leur hybridité. Elles échappent en ce sens aux critères d'identification des catégories traditionnelles de la mobilité spatiale [19]. Néanmoins, un certain nombre de recherches questionnent ces nouvelles formes de mobilité. La longue distance et la longue durée ne sont pas les seules formes de ces nouvelles mobilités : voyages fréquents et bi-résidentialité en font partie également. Jansson estime en Suède à $5 \%$ de la population active la part des pendulaires à la semaine [39]. En Europe, environ $5 \%$ des actifs européens ont des pratiques multilocales pour des raisons professionnelles (bi-résidentialité, voyages fréquents ou relations de couple à distance) qui se déclinent par plus de 60 nuits passées hors du domicile principal [4].

Dans la lignée d'une conception de la mobilité issue de l'École de Chicago [40, 38], nous définissons la mobilité comme : "l'intention, puis la réalisation d'un franchissement de l'espace géographique impliquant un changement social ». L'originalité de l'approche consiste à partir de cette définition générale à concentrer les investigations sur les intentions en matière de mobilité et le passage à l'acte de se déplacer, plus que sur les déplacements proprement dits. C'est ainsi que dans cette approche, chaque personne ou groupe se caractérise par des propensions plus ou moins prononcées à se mouvoir dans l'espace géographique, économique et social. Celles-ci constituent dès lors un ensemble d'aptitudes conceptualisé par la notion de «motilité », en référence à l'acception de ce terme en biologie.

À l'échelle de la personne, la motilité se définit comme : « l'ensemble des caractéristiques propres à un acteur qui permettent d'être mobile, c'est-à-dire les capacités physiques, le revenu, les aspirations à la sédentarité ou à la mobilité, les conditions sociales d'accès aux systèmes techniques de transport et de télécommunication existants, les connaissances acquises, comme la formation, le permis de conduire, l'anglais international pour voyager, etc. » [41]. La motilité se réfère donc aux conditions sociales d'accès (les conditions auxquelles il est possible d'utiliser l'offre au sens large), aux savoirs et compétences (que nécessite l'usage de cette offre) et aux projets de mobilité (l'utilisation effective de l'offre permet de les concrétiser).

La motilité est nécessairement située et reliée à l'espace. Chaque territoire offre un champ des possibles spécifique en matière de mobilité et définit ainsi un potentiel d'accueil aux projets de mobilité. Le potentiel d'accueil d'un territoire est composé de toute une série d'ingrédients : 1. Les réseaux disponibles, leur développement, leurs performances respectives et leurs conditions d'accès : réseaux routiers, autoroutiers, ferroviaires, plateformes aéroportuaires, équipement du territoire en télécommunications. 2. L'espace et l'ensemble de ses configurations territoriales : configurations urbaines, centralités fonctionnelles, territoires institutionnels, etc. 3. Le marché de l'emploi, soit les possibilités de formation et d'emploi et le taux de chômage. 4. Les institutions et lois régissant d'une manière ou d'une autre les activités humaines, par exemple la politique familiale, les aides à la propriété et au logement, la politique d'immigration. 5. L'ensemble des rapports sociaux et des modèles de réussite proposés par une société et les épreuves auxquelles elle soumet les différents acteurs qui la composent.

L'intérêt de la motilité pour décrire et comprendre les pratiques de grande mobilité tient au fait que le monde contemporain se caractérise par une injonction à la mobilité $[42,43]$, tout particulièrement dans la sphère professionnelle, et que tous les individus n'apparaissent pas armés de la même manière pour faire face à cette injonction. La capacité à être mobile est devenue une ressource essentielle pour l'insertion socioprofessionnelle, voire un capital [44], et plusieurs concepts cherchent à la mesurer, tant en géographie avec le capital spatial [45, 46], qu'en sociologie avec le capital en réseau [47] ou avec la motilité [9].

\section{Hypothèses et méthodes}

Au regard des éléments présentés dans la partie théorique, nous visons dans cet article à tester la pertinence du concept de motilité pour décrire la diversité des situations des personnes face à la grande mobilité. Pour ce faire et en lien avec l'état des savoirs sur la question, trois hypothèses seront testées : 
- la première de nos hypothèses est que les différences de motilité permettent de décrire, de comprendre et d'expliquer la situation des personnes face à la grande mobilité ;

- dans la lignée des travaux sur les habitudes liées aux comportements de mobilité, nous faisons ensuite l'hypothèse que la motilité est, à l'échelle de chaque personne, très stable dans le temps ;

- nous faisons finalement l'hypothèse que les personnes les plus motiles sont par voie de conséquence les plus mobiles, et que réciproquement, les moins motiles sont les moins mobiles.

Dans la mesure où la grande mobilité est un phénomène qui échappe en bonne partie aux enquêtes de mobilité et de déplacement traditionnelles, l'utilisation d'enquêtes ad hoc est nécessaire pour travailler sur ces pratiques. Nous nous basons ainsi sur les données de la seconde phase de la recherche JobMob ${ }^{1}$ portant sur l'Allemagne, l'Espagne, la France et la Suisse. Conduite en partenariat avec plusieurs équipes européennes, en 2007 puis en 2011, cette recherche a pour objectif général de questionner la manière dont s'articulent les grandes mobilités pour motif professionnel et la vie de famille. Plusieurs formes de mobilité sont prises en compte dans cette enquête : pendularité de longue durée, définie par le fait de se déplacer plus de 2 heures par jour (aller-retour) pour se rendre au travail, au minimum 3 fois par semaine ; absents du domicile plus de 60 nuits par an pour des motifs professionnels (ce peut être des bi-résidents ou des voyageurs fréquents); relations de couple à distance pour des motifs professionnels.

La base de données, constituée avec la méthode CATI (Computer-Assisted Telephone Interview), contient 1735 individus, enquêtés en 2007 puis en 2011, il s'agit donc une enquête de panel. La dimension longitudinale permet de mesurer les changements dans les comportements des individus et de tenter de les expliquer. L'échantillon a été construit en 2007 pour être représentatif de la population des 25-54 ans. À noter qu'un coefficient de pondération a été construit en 2011 pour limiter les effets de l'attrition (perte d'effectif) entre les deux vagues d'enquêtes. Par ailleurs, des données sur le taux de chômage dans la région de domicile (niveau NUTS 2) ${ }^{2}$ et sur le degré d'urbanisation (de la commune de domicile) ont été utilisées pour caractériser les contextes spatiaux. Les communes des 4 pays sont ainsi classées selon une différenciation ${ }^{3}$ en 3 types : fortement, moyennement et faiblement urbanisées.

\footnotetext{
${ }^{1}$ Job mobilities and family lives, http://www.jobmob-and-famlives.eu/ ${ }^{2}$ Données ESPON.

${ }^{3}$ Pour plus d'informations relatives aux degrés d'urbanisation : $\mathrm{http} / / /$ ec.europa.eu/eurostat/ramon/miscellaneous/index.cfm?Target Url=DSP_DEGURBA
}

\section{Comment mesurer la motilité ?}

La typologie de la motilité, que nous allons présenter ici, est l'élément central autour duquel cet article est construit. La typologie proposée ne mesure pas la motilité en général, mais la motilité en lien avec la grande mobilité. En effet, l'objet de notre recherche nécessite une exploration en profondeur de la capacité des personnes à se déraciner et s'enraciner ailleurs, de leurs aptitudes à la réversibilité et d'une manière plus générale de leurs aptitudes à gérer la distance et la vitesse.

La typologie se base sur une quinzaine de variables relatives aux trois dimensions de la motilité (accès, compétences et projets) (Tableau 1). La première dimension est constituée d'une part des accès aux infrastructures de transport (autoroute, gare régionale/RER et lignes à grande vitesse, et aéroport) et d'autre part des accès à des équipements facilitant la mobilité (voiture, internet et ordinateur portable). Les compétences de mobilité sont mesurées à travers la capacité à s'orienter dans l'espace et à lire une carte, les compétences linguistiques, ainsi que la capacité à considérer un déménagement comme une opportunité. Ces compétences traduisent ainsi différents aspects des aptitudes nécessaires à la grande mobilité, tels que la maîtrise des temps et des espaces des déplacements, la capacité à s'approprier des espaces inconnus, à s'ancrer dans des territoires nouveaux ou encore la capacité à communiquer dans un environnement de travail international. Finalement, la dimension projet est ici abordée à travers les dispositions des individus à pratiquer différentes formes de (grande) mobilité pour des motifs professionnels, telles que la pendularité de longue durée ou encore la migration ${ }^{4}$. Techniquement, une analyse factorielle a été réalisée sur ces différentes variables dans un premier temps. À partir des six axes factoriels retenus (dont la valeur propre était supérieure à $1^{5}$ ) et pondérés par leur valeur propre, une classification hiérarchique (méthode de Ward) a été appliquée. Une typologie de la motilité en 6 groupes a ainsi été créée (voir partie suivante). Par ailleurs, travailler avec des données longitudinales impose l'utilisation de méthodes particulières. En l'occurrence, nous souhaitions disposer de typologies similaires pour les deux années considérées (2007 et 2011). Ainsi, nous avons utilisé une méthode dite multidates [49]. Pour cela, nous avons compilé les données des deux enquêtes en une base unique, en considérant artificiellement les individus de 2007 et 2011 comme différents. Sur cette base, nous avons construit une typologie avec une méthode statistique classique (voir plus haut). Chaque individu se retrouve donc associé à deux groupes de motilité, un pour 2007 et un pour 2011 ; ces deux

\footnotetext{
${ }^{4}$ Pour plus d'informations relatives au choix des variables voir (48)

${ }^{5} 59 \%$ de la variance.
} 
groupes pouvant être ensuite comparés. Cette technique permet d'avoir des groupes relativement stables dans le temps, dans la mesure où une seule typologie a été créée.

Pour caractériser les groupes de motilité, nous avons eu recours à plusieurs modèles de régression logistique, nous permettant de préciser la composition sociodémographique des groupes, l'ancrage local des personnes (vie à l'étranger, déménagements antérieurs, type d'habitat, attachement au logement actuel, à la ville ou au pays de résidence, etc.) ainsi que leur rapport à la grande mobilité (pratiques actuelles ou passées, refus et non-confrontation).

\section{La motilité pour la grande mobilité. Présentation des résultats}

\section{Quels profils de motilité ?}

À travers la typologie présentée ci-dessus se dégagent six profils de motilité (peu motiles, réticents à bouger, prêts à bouger, réversibles, non-réversibles et très motiles). La dénomination des groupes renvoie aux caractéristiques fortes de leur potentiel de grande mobilité, en regard des différentes dimensions et variables qui le composent. Ainsi, les réversibles et les non-réversibles ne pratiquent pas nécessairement les formes de mobilités éponymes, mais ce nom leur a été donné en référence à leur préférence pour ces formes de mobilité. À ce stade de l'analyse, c'est bien le potentiel de mobilité et non la mobilité effective qui nous intéresse.

Le premier groupe issu de notre typologie ${ }^{6}$ est composé de personnes dont la motilité peut être considérée comme faible $(24,1 \%$ de l'échantillon). Bien que leurs accès aux systèmes de transports soient proches de la moyenne, leur accès aux équipements personnels facilitant la mobilité est inférieur, particulièrement concernant la possession d'un ordinateur portable (Tableau 1). Toutefois, c'est bien par leurs compétences, très faibles en langues notamment, et leur disposition à la mobilité, faible également, que les peu motiles se caractérisent. Les plus âgés sont surreprésentés dans ce groupe des personnes peu motiles. Cela s'explique par un effet de position dans le cycle de vie et par un effet de génération. Ainsi, avec l'âge, le potentiel de mobilité peut se réduire en raison de capacités physiques déclinantes ou de difficultés à s'adapter et à tirer avantage des nouvelles technologies, telles qu'Internet, smartphones, portables ou autres. Par ailleurs, les individus cherchent avec le temps à stabiliser leur cadre de vie et sont par conséquent moins prêts à faire face à de grands changements [18]. Corroborant

\footnotetext{
${ }^{6}$ Pour éviter les redondances, seuls les résultats de la typologie pour l'année 2011 sont présentés. Les évolutions entre 2007 et 2011 sont discutées ci-dessous.
}

l'idée d'une étape dans le parcours de vie où la mobilité est moins envisageable, on constate une plus grande part de propriétaires chez les peu motiles. Néanmoins, cette réticence face à la mobilité s'inscrit dans le long terme puisque les peu motiles ont plus de chances d'avoir déjà refusé un poste qui impliquerait de la grande mobilité. Dans le même ordre d'idée, ils sont proportionnellement moins à avoir eu à se poser la question. Pour beaucoup d'entre eux, aucune opportunité du genre ne s'est présentée. Cette faible confrontation à la grande mobilité de la part des peu motiles renvoie à certains secteurs du marché de l'emploi, axés principalement sur le local, à des carrières professionnelles effectuées en partie dans des périodes où l'emploi était moins flexible ou encore à un attachement plus marqué aux territoires proches du lieu de domicile. En effet, ces personnes se déclarent particulièrement attachées à leur cadre de vie, qu'il s'agisse de leur ville ou de leur pays de résidence. Par ailleurs, on note un lien entre cette faible motilité et le revenu (prépondérance des bas revenus). Ce résultat est cohérent avec de nombreuses études citant le revenu comme un des facteurs importants du potentiel de mobilité $(47,9,50)$.

Le groupe des très motiles (11,8\% de l'échantillon) s'oppose par essence au premier, celui des peu motiles. Les personnes qui le composent disposent de très bons accès et compétences et sont prêtes à être mobiles, quelle que soit la forme de cette mobilité. Même la bi-résidentialité, très peu prisée des autres groupes (mis à part les prêts à bouger) est envisageable pour eux. Les personnes issues de ce groupe sont avant tout des hommes, jeunes, sans enfant (seuls ou en couple) et très peu d'entre eux sont propriétaires. Cette disposition à la mobilité très élevée s'accompagne par un très faible attachement au pays de résidence, par des expériences de vie à l'étranger et de grande mobilité passées. Ils ont par ailleurs très peu de chances d'avoir déjà refusé un emploi qui aurait impliqué de longs trajets ou des nuits hors domicile. Les très motiles illustrent un mode de vie qui ne se limite pas au pays de résidence. Parallèlement, les contraintes familiales ne les retiennent pas (ou peu) lorsqu'il s'agit de saisir des opportunités d'emploi où qu'elles se trouvent et quelles que soient les formes de mobilité qu'elles impliquent.

Au-delà d'une description ordinale de la motilité, le groupe des réticents à bouger (22,6\% de l'échantillon) se démarque par des dispositions faibles à la mobilité, mais des compétences élevées. Les réticents à bouger sont très bien formés et sont peu à déclarer de faibles revenus. Ce découplage entre compétences/accès et disposition à la mobilité est particulièrement intéressant : il apparait que le désir d'immobilité concerne également les mieux armés en compétences de mobilité ou les mieux formés. Par ailleurs, ce rejet de la grande mobilité s'inscrit dans le long terme. D'une part, ils ont plus de chances d'avoir déjà refusé un 


\begin{tabular}{|c|c|c|c|c|c|c|c|}
\hline & Peu motiles & $\begin{array}{l}\text { Réticents à } \\
\text { bouger }\end{array}$ & $\begin{array}{l}\text { Prêts à } \\
\text { bouger }\end{array}$ & Réversibles & Non-réversibles & $\begin{array}{l}\text { Très } \\
\text { motiles }\end{array}$ & $\begin{array}{l}\text { Ensemble } \\
\text { de la } \\
\text { population }\end{array}$ \\
\hline & $24,1 \%$ & $22,6 \%$ & $13,6 \%$ & $14,9 \%$ & $13,1 \%$ & $11,8 \%$ & $100 \%$ \\
\hline \multicolumn{8}{|c|}{ Accès contextuels (à une distance de $X$ minutes du lieu de domicile) } \\
\hline Autoroute $(20 \mathrm{~min})$. & 0,87 & 0,85 & 0,80 & 0,95 & 0,97 & 0,95 & 0,89 \\
\hline $\begin{array}{l}\text { Train régional (RER) } \\
\text { (20 min.) }\end{array}$ & 0,90 & 0,86 & 0,82 & 0,98 & 10,00 & 0,88 & 0,90 \\
\hline $\begin{array}{l}\text { Ligne à grande vitesse } \\
(\mathrm{TGV})(20 \mathrm{~min} .)\end{array}$ & 0,59 & 0,58 & 0,48 & 0,70 & 0,57 & 0,67 & 0,60 \\
\hline Aéroport (45 min.) & 0,64 & 0,58 & 0,63 & 0,63 & 0,85 & 0,72 & 0,66 \\
\hline \multicolumn{8}{|l|}{ Accès personnels } \\
\hline Ordinateur portable & 0,48 & 0,64 & 0,55 & 0,73 & 0,77 & 0,75 & 0,63 \\
\hline Internet & 0,89 & 0,96 & 0,88 & 0,98 & 10,00 & 0,96 & 0,94 \\
\hline Voiture & 0,90 & 0,95 & 0,89 & 0,81 & 0,98 & 0,91 & 0,91 \\
\hline \multicolumn{8}{|l|}{ Compétences } \\
\hline $\begin{array}{l}\text { Nombre de langues } \\
\text { parlées }\end{array}$ & 0,30 & 0,70 & 0,37 & 0,70 & 0,69 & 0,67 & 0,56 \\
\hline Maîtrise de l'anglais & 0,00 & 0,96 & 0,09 & 0,95 & 10,00 & 0,91 & 0,61 \\
\hline $\begin{array}{l}\text { Capacité à lire une carte et } \\
\text { se repérer dans l'espace }\end{array}$ & 0,62 & 0,71 & 0,74 & 0,65 & 0,85 & 0,73 & 0,70 \\
\hline $\begin{array}{l}\text { Capacité à considérer un } \\
\text { déménagement (à plus } \\
\text { de } 50 \mathrm{~km} \text { du domicile) } \\
\text { comme une opportunité }\end{array}$ & 0,54 & 0,56 & 0,60 & 0,33 & 0,94 & 0,80 & 0,61 \\
\hline \multicolumn{8}{|l|}{ Disposition à la mobilité } \\
\hline $\begin{array}{l}\text { Disposition à la migration } \\
\text { interrégionale }\end{array}$ & 0,13 & 0,14 & 0,46 & 0,19 & 0,49 & 0,80 & 0,31 \\
\hline $\begin{array}{l}\text { Disposition à la migration } \\
\text { internationale }\end{array}$ & 0,07 & 0,09 & 0,42 & 0,18 & 0,43 & 0,69 & 0,26 \\
\hline $\begin{array}{l}\text { Disposition à la } \\
\text { pendularité de longue } \\
\text { durée }\end{array}$ & 0,28 & 0,11 & 0,81 & 0,54 & 0,32 & 0,84 & 0,42 \\
\hline $\begin{array}{l}\text { Disposition à la } \\
\text { bi-résidentialité }\end{array}$ & 0,06 & 0,02 & 0,62 & 0,11 & 0,06 & 0,76 & 0,22 \\
\hline $\begin{array}{l}\text { Disposition aux voyages } \\
\text { fréquents }\end{array}$ & 0,17 & 0,15 & 0,76 & 0,59 & 0,53 & 0,93 & 0,44 \\
\hline
\end{tabular}

emploi qui impliquait d'être grand mobile. D'autre part, les réticents à bouger ont durant leur carrière une probabilité plus faible d'avoir été pendulaires de longue durée ou absents du domicile, mais ont par contre plus de chances d'avoir déjà migré (éventuellement pour éviter de longs déplacements ou des nuits hors domicile). Leur position sociale semble leur permettre d'être en accord avec leurs projets de mobilité qu'il s'agisse d'être grand mobile, de le devenir ou d'arrêter de l'être, mais aussi de refuser la mobilité. Dans ce groupe comme dans celui des personnes peu motiles, les femmes sont surreprésentées.

Diamétralement opposés aux réticents à bouger, les prêts à bouger (13,6 \% de l'échantillon) combinent des compétences limitées (pour les langues principalement) et 
des accès inférieurs à la moyenne avec une forte disposition à la mobilité. Si les premiers renvoyaient l'image d'une immobilité choisie, les prêts à bouger semblent quant à eux, contraints à la mobilité par un contexte économique voire social défavorable. Leurs très faibles compétences linguistiques et le fait que peu d'entre eux aient déjà déménagé à plus de $50 \mathrm{~km}$ de chez eux pour des raisons professionnelles ne les empêchent pas de se déclarer prêts à migrer ou à voyager fréquemment. Le contexte de vie peu favorable de ces individus s'illustre notamment à travers le type de logement : les prêts à bouger ont une probabilité près de trois fois plus grande d'être hébergés chez des proches (amis ou famille) sans payer de loyer, que d'être propriétaires de leur logement. Les hommes, les individus vivants seuls, les personnes dans la quarantaine, et celles disposant de formations intermédiaires ont une probabilité plus grande de faire partie de ce groupe.

Le quatrième groupe issu de notre typologie, les réversibles (14,9\% de l'échantillon), est composé de personnes qui disposent de bons accès aux systèmes de transport et aux équipements facilitant la mobilité, ainsi que de bonnes compétences et qui déclarent privilégier les formes de mobilité dites réversibles, telles que la pendularité de longue distance voire les voyages fréquents. La composition de ce groupe semble être assez hétérogène : il n'y a pas de tendance claire au niveau de l'âge, du genre et du niveau de formation. Toutefois, on note que les réversibles ont plus tendance à avoir des enfants et que les hauts salaires sont bien représentés dans ce groupe. La disposition à la réversibilité actuelle de ces individus n'exclut pas de facto des mobilités irréversibles anciennes : ainsi, la majorité d'entre eux ont déjà vécu à l'étranger ou déménagé pour le travail. Leurs dispositions semblent liées à un attachement certain (modéré voire fort) à leur ville et leur pays de résidence.

Les non-réversibles ( $13 \%$ de l'échantillon) sont caractérisés par de très bons accès et compétences (les meilleurs de l'échantillon) et par des dispositions à la mobilité plus tournées vers les formes irréversibles à l'instar de la migration interrégionale et internationale, mais aussi vers les voyages fréquents, que vers la pendularité de longue durée ou la bi-résidentialité. Le nom de ce groupe renvoie à la faible disposition de ces individus envers les mobilités réversibles régulières. Ces non-réversibles sont principalement des hommes, jeunes et bien formés ; peu d'entre eux disposent de bas salaires. Cette forte disposition au déménagement s'accompagne d'attaches plutôt faibles au cadre de vie (logement, ville ou pays). La prépondérance de locataires parmi les non-réversibles va dans la même direction. À noter également que les familles monoparentales (avec à leur tête des femmes principalement) ont une probabilité plus élevée de faire partie de ce groupe. Ainsi, pour les personnes qui élèvent seules leur(s) enfant(s), le déménagement dans une autre région ou un autre pays paraît être une solution plus envisageable que de longs trajets quotidiens ou des absences du domicile, et ceci malgré les fortes implications en termes de changement et de déracinement qu'un déménagement lointain peut impliquer.

La motilité permet finalement de mettre en évidence une double distinction entre les individus vis-à-vis de la grande mobilité. La première concerne le couplage et découplage qui apparaît entre les compétences et les accès d'un côté et les dispositions à la mobilité d'un autre côté. Quatre groupes se trouvent aux deux extrêmes, les peu motiles et les réticents à bouger (faible disposition), les prêts à bouger et les très motiles (forte disposition). La seconde distinction qui apparaît dans les potentialités individuelles concerne les mobilités réversibles et irréversibles. Les groupes des réversibles et non-réversibles permettent ainsi de montrer que certaines personnes sont prêtes à déménager pour éviter les pendularités tandis que d'autres vont précisément défendre la position inverse, à savoir être prêt à effectuer de longs trajets chaque jour pour éviter de déménager... Cette distinction fait écho aux résultats déjà discutés d'une substitution entre déménagements et pendularités de longue distance [33, 34]. Les diverses variables utilisées pour caractériser ces groupes tels que l'âge, le genre, le revenu ou le cadre de vie sont éclairantes sur la manière dont les individus sont aptes et disposés à se confronter à des expériences de grande mobilité. L'importance des éléments liés au ménage (vie de couple, présence d'enfants, etc.) invite à une considération plus large de la manière dont le potentiel de (grande) mobilité se construit à l'échelle du ménage (notamment en fonction des dispositions des différents membres du ménage, des ancrages de chacun, de l'activité professionnelle du conjoint et l'éloignement éventuel de son lieu de travail, etc.). Cette analyse dépasse néanmoins le cadre de cet article.

\section{La motilité selon le territoire de résidence}

La deuxième étape de l'analyse des groupes de motilité vise à comprendre l'influence des contextes spatiaux sur la motilité. Dans ce but, trois indicateurs sont utilisés : le pays de résidence, le degré d'urbanisation de la commune de résidence et le taux de chômage dans la région de résidence (niveau NUTS2) ${ }^{7}$.

Le pays de résidence a une influence certaine sur les groupes de motilité. Si l'on se concentre sur les personnes

\footnotetext{
${ }^{7}$ Seul le tableau 2 concernant la répartition des groupes de motilité selon le pays de résidence est présenté.
} 


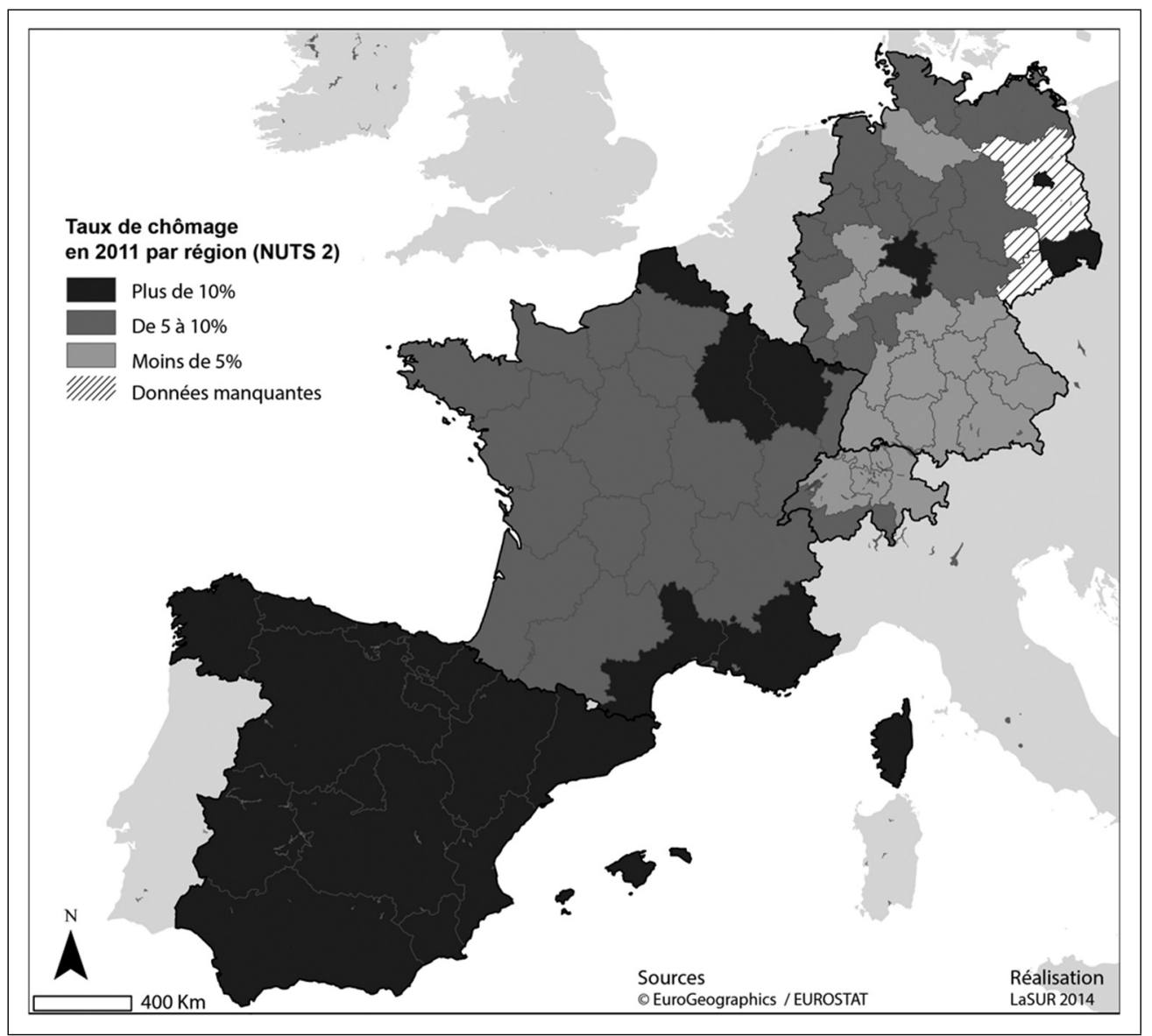

Fig. 1 Taux de chômage par région en 2011 (NUTS 2)

Tableau 2 Répartition des groupes de motilité selon le pays de résidence en 2011

France Allemagne Espagne Suisse

\begin{tabular}{lrrrr}
\hline Peu motiles & $27 \%$ & $13 \%$ & $33 \%$ & $24 \%$ \\
Réticents à bouger & $23 \%$ & $28 \%$ & $7 \%$ & $31 \%$ \\
Prêts à bouger & $15 \%$ & $7 \%$ & $29 \%$ & $5 \%$ \\
Réversibles & $14 \%$ & $21 \%$ & $6 \%$ & $19 \%$ \\
Non-réversibles & $15 \%$ & $16 \%$ & $6 \%$ & $15 \%$ \\
Très motiles $^{8} \%$ & $6 \%$ & $16 \%$ & $20 \%$ & $6 \%$ \\
Total $^{8}$ & $100 \%$ & $100 \%$ & $100 \%$ & $100 \%$ \\
\hline
\end{tabular}

Tableau croisé, Population totale, 29-58 ans en 2011, JobMob II, pondération panel nation analysis.

qui ont de faibles dispositions à toutes les formes de grande mobilité (groupes des peu motiles et des réticents à bouger), elles apparaissent comme davantage représentées en Suisse

${ }^{8}$ Chi2 $=329,627, \mathrm{p}<0,001$.
(55\%) et en France (50\%), qu'en Allemagne et en Espagne (autour de $40 \%$ ). Pour autant, ces personnes sont dans deux situations très différentes vis-à-vis de ce refus d'être grand mobile. En effet, certains ont de faibles accès et compétences (les peu motiles), alors que les autres en ont de bons (les réticents à bouger). C'est en Suisse et en Allemagne que l'on rencontre le plus ces derniers. Ce résultat s'accorde avec la tendance plus générale qui veut que les personnes ayant les meilleurs accès et compétences de mobilité soient davantage représentées dans ces deux pays. Quant à la surreprésentation des prêts à bouger parmi les Espagnols, elle renvoie très certainement à la situation économique difficile du pays.

Les taux de chômage sont très différents selon les pays et plus précisément les régions considérées (Fig. 1). Ainsi, plus le taux de chômage dans la région de résidence est élevé, plus la proportion de peu motiles, de très motiles et de prêts à bouger augmente. Les différences les plus importantes concernent ce dernier groupe : alors qu'ils représentent que $4.5 \%$ des individus des régions où le taux de chômage est de moins de $5 \%$, ils comptent pour plus d'un quart de la population dans les régions les plus touchées par le chômage ! A contrario, les autres groupes de motilité 


\begin{tabular}{|lll|}
$\begin{array}{l}\text { Tableau } 3 \text { Surreprésentation des groupes de motilité selon le } \\
\text { degré d'urbanisation et la santé économique du territoire }\end{array}$ \\
\hline & $\begin{array}{l}\text { Bonne santé } \\
\text { économique des } \\
\text { territoires }\end{array}$ & $\begin{array}{l}\text { Mauvaise santé } \\
\text { économique } \\
\text { des territoires }\end{array}$ \\
\hline Forte urbanité & $\begin{array}{l}\text { Réversibles } \\
\text { Non-réversibles } \\
\text { Réticents à bouger }\end{array}$ & $\begin{array}{l}\text { Très motiles } \\
\text { Prêts à bouger } \\
\text { Peu motiles }\end{array}$ \\
\hline
\end{tabular}

connaissent la relation inverse, puisque leur proportion diminue lorsque le taux de chômage augmente. Bien que la relation entre le degré d'urbanisation et la motilité soit moins forte que celles avec le pays de résidence et le taux de chômage, nous pouvons observer que les deux groupes les moins disposés à la grande mobilité ont tendance à vivre dans des zones moins urbanisées. Les très motiles et les réversibles, eux, se trouvent principalement dans les communes les plus centrales des grandes agglomérations. Les non-réversibles se localisent à parts égales dans les différents types de communes. Ainsi, les très motiles, que l'analyse sociodémographique ne pouvait décrire précisément, apparaissent comme majoritairement urbains et vivent, en partie du moins, dans des zones touchées par le chômage comme Madrid, Palma de Majorque, Berlin ou Marseille par exemple. Le tableau 3 ci-dessus présente de manière schématisée les groupes surreprésentés dans les zones selon l'urbanisation et la santé économique de celles-ci.

$\mathrm{Au}$ regard de l'ensemble des résultats présentés dans cette section grâce à la mise en place d'une typologie de la motilité, nous concluons sur la validité de la première hypothèse. Les groupes de motilité sont certes liés aux variables sociodémographiques usuelles telles que l'âge, le genre, le niveau d'éducation, le revenu, etc., mais ils permettent de mettre en évidence une relation plus complexe et plus intime à la mobilité. C'est ainsi au cœur des arbitrages entre vie privée et vie professionnelle que l'on peut comprendre pourquoi le potentiel des personnes est orienté vers des pendularités quotidiennes, ou vers des déménagements ou des migrations, vers des formes variées de grande mobilité, ou vers un refus de ces pratiques. L'arbitrage en question sera alors nécessairement orienté par les opportunités ou les contraintes propres au territoire de résidence. Lorsque le chômage augmente dans une région par exemple, le découplage se renforce entre accès et compétences d'un côté, et disposition de l'autre, ce qui se traduit par une augmentation très forte du groupe des prêts à bouger.

\section{Stabilité et évolutivité de la motilité}

Après avoir considéré et décrit la motilité des individus en 2011, c'est ici la question de l'évolution de ses différentes modalités, mais aussi l'évolution de la distribution des groupes de motilité qui nous intéresse. Premièrement, les trois dimensions de la motilité évoluent selon des temporalités différentes. Si les projets de mobilité ou les équipements personnels (par ex. l'accès aux nouvelles technologies) évoluent à court terme, les compétences (linguistiques en particulier) chez les adultes sont dans une grande mesure figées. En effet, dans l’Union européenne, seule une infime part des personnes continuent d'apprendre de nouvelles langues à l'âge adulte [51]. De même, les infrastructures de transports n'évoluent que très lentement ${ }^{9}$. Entre les deux vagues, l'accès aux ordinateurs portables et à Internet a lui clairement augmenté. En revanche, les dispositions à la mobilité évoluent différemment selon le type de mobilité considéré : la disposition aux mobilités réversibles augmente alors que les formes plus irréversibles telles que la migration interrégionale et internationale sont moins plébiscitées en 2011. De manière contre-intuitive, il apparaît que les compétences telles que la capacité à lire une carte et à s'orienter dans l'espace ont diminué entre les deux vagues, mettant à mal, à première vue, l'idée d'un capital de mobilité se renforçant avec l'expérience. Toutefois, observée de plus près, la baisse n'est significative que pour les personnes non-mobiles en 2007 et en 2011. Dans l'ensemble, il apparaît que les variables composant la motilité évoluent moins, et/ou de manière non-significative, chez les personnes mobiles.

La distribution des différents types de motilité dans la population reste globalement assez stable entre 2007 et 2011. Certains groupes connaissent cependant des évolutions plus marquées. C'est le cas notamment du groupe des réversibles, qui augmente significativement entre 2007 et 2011 : les individus composant ce groupe se définissent notamment par une disposition à la mobilité privilégiant avant tout des formes de mobilité réversibles, telles que la pendularité de longue durée (quotidiennement) et les voyages fréquents. Alors qu'ils représentaient en 2007 moins de $10 \%$ de l'échantillon, ils comptent quatre ans plus tard pour près de $15 \%$ de la population. Il convient de rappeler qu'il s'agit lors des deux vagues des mêmes

\footnotetext{
${ }^{9}$ Suivant cette logique, il a été décidé de ne pas reposer certaines questions dans le deuxième volet de l'enquête, à savoir les questions relatives aux compétences linguistiques, ainsi que celles concernant les accès contextuels. Pour ces variables, les valeurs de 2007 ont donc été utilisées, sauf en Suisse et en France où les questions portant sur les accès contextuels ont tout de même été à nouveau posées. Les évolutions des accès contextuels dans ces deux pays entre 2007 et 2011 sont pour la plupart insignifiantes, confirmant l'hypothèse d'une grande stabilité dans le temps.
} 
Tableau 4 Évolution des types de motilité par pays entre 2007 et 2011 (en points)

\begin{tabular}{|c|c|c|c|c|c|}
\hline & France & Allemagne & Espagne & Suisse & Total \\
\hline Peu motiles & -6 & +3 & -7 & -1 & -3.4 \\
\hline Réticents à bouger & -3 & -4 & -8 & -1 & -3.1 \\
\hline Prêts à bouger & +6 & -3 & +7 & +2 & +2.6 \\
\hline Réversibles & +7 & +9 & -2 & +7 & +5.1 \\
\hline Non-réversibles & +2 & -3 & -1 & -3 & -1.1 \\
\hline Très motiles & -6 & -1 & +11 & -4 & 0 \\
\hline
\end{tabular}

individus et que ceux-ci ont vieilli de quatre ans : cette progression des réversibles fait écho à l'ancrage croissant des populations au fil de l'avancement dans le cycle de vie. Cette tendance est commune à tous les pays sauf en Espagne, où ce groupe perd en importance. Dans ce pays, les ancrages de manière générale semblent devenir plus difficiles à conserver. Ceci se confirme à travers l'analyse des individus les moins disposés à être mobile. En effet, leur proportion diminue dans tous les pays, mais plus fortement en Espagne. Cette tendance suggère ainsi que le refus de toute forme de mobilité semble être de plus en plus difficile pour garantir l'emploi et beaucoup sont contraints d'envisager déménagements, bi-résidentialité ou pendularité de longue durée en raison notamment d'un contexte économique défavorable. La pendularité de longue durée apparaît ainsi comme l'une des plus « acceptables », au sens où elle garantit un certain ancrage autour du logement. En Espagne, toutefois, cette dernière semble être une concession insuffisante. Si le groupe des très motiles continue à représenter une part similaire de la population (à noter une très forte augmentation en Espagne et une baisse dans les autres pays), les prêts à bouger, l'autre groupe particulièrement disposé à se déplacer, connaît une augmentation de 2,6 points. Ainsi, le décalage entre compétences/accès faibles et disposition à la mobilité élevée se renforce entre les deux vagues d'enquête, avant tout dans les deux pays les plus touchés par la crise économique, l'Espagne et la France.

De ces analyses, trois grandes tendances se dessinent quant aux évolutions de la motilité dans le temps et permettent, à quelques nuances près, d'invalider l'hypothèse 2 concernant la stabilité dans le temps, pour chaque personne, de la motilité.

Premièrement, les changements de type de motilité entre la vague 1 et la vague 2 répondent dans la majorité des cas à des réajustements dans les projets de mobilité. Les individus repensent dans le temps de manière marquée leur disposition à la mobilité. Toutefois, les passages d'une classe à l'autre sont conditionnés par le niveau de compétences et d'accès des individus : la quasi-totalité des évolutions se fait parmi des individus qui partagent des compétences et des accès similaires, suggérant une très faible évolution dans le temps, du moins à court terme, de ces deux dimensions.

Deuxièmement, il apparaît que le refus de toute forme de grande mobilité (ou l'immobilité) devient de plus en plus difficile. Les mobilités réversibles les moins impliquantes socialement parlant, à l'instar de la pendularité, sont ainsi à un niveau européen de plus en plus privilégiées aux dépens de la mobilité résidentielle interrégionale et internationale. Ces deux tendances indiquent un renforcement de l'ancrage résidentiel. Toutefois, en Espagne, la pendularité de longue durée et l'immobilité semblent insuffisantes ou inconciliables avec un maintien sur le marché de l'emploi.

Troisièmement, les changements de groupe de motilité sont très fortement influencés par le contexte national (et économique). Ceci se confirme d'une part par une faible évolution de la composition sociodémographique des groupes. D'autre part, les projets de mobilité sont influencés par les changements familiaux (arrivée d'un enfant, séparation/mise en couple), mais aussi par les changements dans la situation économique des individus (évolution du taux de travail ou du salaire). Ainsi, la situation économique à la fois individuelle et contextuelle apparaît ainsi comme cruciale dans l'évolution des projets de mobilité et plus généralement de la motilité.

\section{Être motile, pour être mobile?}

Jusqu'ici, l'apport du concept de motilité pour expliquer et comprendre la grande mobilité n'a été abordé qu'indirectement. Cette partie vise justement à répondre à cette interrogation en questionnant l'influence de la motilité (et des variables sociodémographiques) sur la grande mobilité. Pour cela, il est toutefois utile en premier 
lieu de s'intéresser aux pratiques de grande mobilité dans la population en général, mais aussi de mettre en lumière les différences entre pays, degrés d'urbanisation et santé économique des territoires ${ }^{10}$.

$10 \%$ environ des individus de notre échantillon sont considérés comme grands mobiles, toutes catégories confondues. C'est en Allemagne que l'on en trouve le plus, suivie de la Suisse, la France et l'Espagne. La santé économique des deux premiers pays semble être en lien avec la part plus élevée de grande mobilité : en effet, il apparaît que plus le chômage est élevé, moins le recours à la mobilité est important (particulièrement en ce qui concerne la pendularité de longue durée). La part des pendulaires de longue durée parmi les grands mobiles est la plus élevée en Suisse avec $71 \%$. En France et en Allemagne, elle se situe autour des $50 \%$, alors qu'en Espagne, elle n'est que de $31 \%$. Finalement, les pratiques de mobilité ne sont pas liées au degré d'urbanisation : ainsi, la part de grands mobiles est similaire tant dans les centres urbains que dans les communes moyennement et faiblement urbanisées.

Il est intéressant de constater que la grande mobilité n'est que peu expliquée par les variables sociodémographiques (Tableau 5). Ni le revenu, ni la formation ou l'âge n'influencent significativement le recours à la grande mobilité. Seuls le genre et la composition du ménage ont un effet : ainsi, les hommes, les personnes seules et les familles monoparentales ont plus de chances d'être mobiles. Le constat est similaire pour la pendularité de longue durée et l'absence du domicile, hormis que l'influence de la structure du ménage est moins décisive.

La motilité a par contre un grand pouvoir explicatif. Lorsque l'on prend en compte toutes les formes de grande mobilité, le groupe des réticents à bouger apparaît comme le groupe qui a la plus faible probabilité d'être mobile (environ 2 fois moins). Il semblerait que leur position sociale et leurs compétences leur permettent d'être en adéquation avec leur volonté, c'est-à-dire d'éviter la grande mobilité. Ils ont d'ailleurs une probabilité encore plus faible d'être grands mobiles que les peu motiles, caractérisés par leur faible potentiel de mobilité. Tel n'est pas le cas des réversibles et des non-réversibles, qui eux sont plus souvent mobiles (entre 1,5 et 2 fois plus de chances). Il est très intéressant de constater que les très motiles, pourtant caractérisés par de très bonnes compétences, de bons accès et une forte disposition à la mobilité n'ont pas une probabilité plus forte d'être grand mobile (toutes formes confondues). En mettant en regard ce résultat avec la composition sociologique du groupe (prépondérance des jeunes) et leurs expériences de grandes mobilités passées, le rapport qu'entretient ce groupe avec la grande motilité pourrait illustrer des carrières

\footnotetext{
$\overline{10}$ Tableaux non présentés.
}

\begin{tabular}{|c|c|c|c|}
\hline & $\begin{array}{l}\text { Mobilité } \\
\text { (tous types) }\end{array}$ & $\begin{array}{l}\text { Pendulaires } \\
\text { de longue } \\
\text { durée }\end{array}$ & $\begin{array}{l}\text { Absents } \\
\text { du } \\
\text { domicile }\end{array}$ \\
\hline Peu motiles & $0,675^{*}$ & 1,155 & $0,151 * *$ \\
\hline Réticents à bouger & $0,496 * *$ & 0,661 & $0,404 *$ \\
\hline Prêts à bouger & 0,98 & 1,110 & $1,948^{*}$ \\
\hline Réversibles & $1,903 * * *$ & $2,487 * * *$ & 1,601 \\
\hline Non-réversibles & $1,491 *$ & 0,670 & $2,811 * * *$ \\
\hline $\begin{array}{l}\text { Très motiles } \\
\text { 29-38 ans (réf.) }\end{array}$ & 1,070 & 0,708 & $1,871^{*}$ \\
\hline $39-48$ ans & 1,388 & 1,269 & 0,764 \\
\hline $\begin{array}{l}49-58 \text { ans } \\
\text { Femme (réf.) }\end{array}$ & 1,172 & 1,290 & 0,901 \\
\hline Homme & $1,675 * *$ & $1,861^{*}$ & $1,981^{*}$ \\
\hline $\begin{array}{l}\text { Pas de formation } \\
\text { post-obligatoire }\end{array}$ & 0,860 & 0,878 & 1,021 \\
\hline $\begin{array}{l}\text { Formation secondaire } \\
\text { (II) (réf.) }\end{array}$ & & & \\
\hline $\begin{array}{l}\text { Formation tertiaire } \\
\text { En couple avec } \\
\text { enfant(s) (réf.) }\end{array}$ & 0,908 & 0,966 & 0,536 \\
\hline Seul & $1,583 *$ & 0,595 & 0,942 \\
\hline En couple sans enfant & 1,101 & 1,023 & 1,182 \\
\hline $\begin{array}{l}\text { Famille } \\
\text { monoparentale }\end{array}$ & $4,054 * * *$ & $3,476^{* * *}$ & 2,232 \\
\hline Salaire moyen (réf.) & & & \\
\hline Bas salaire & 0,930 & 0,856 & 1,113 \\
\hline Haut salaire & 1,127 & 0,959 & 1,265 \\
\hline $\begin{array}{l}\text { Significativité du } \\
\text { modèle }\end{array}$ & 0,000 & 0,002 & 0,000 \\
\hline N (non-pondéré) & 1496 & 1496 & 1496 \\
\hline
\end{tabular}

de moins en moins stables, marquées par des périodes d'emplois courtes (CDD) et successives. En outre, les faibles ancrages locaux des très motiles peuvent illustrer une génération plus internationale, largement ouverte aux possibilités qu'offre un grand marché du travail européen.

Lorsque l'on considère uniquement les pendularités de longue durée, la seule relation significative concerne les réversibles. En effet, ces derniers ont 2,5 fois plus de chances de pratiquer ce type de mobilité. En revanche, à propos des absents du domicile, on constate que les peu motiles (principalement) et les réticents à bouger ne 
sont que très peu concernés (en raison de leurs faibles compétences et accès et/ou de leurs faibles dispositions). A contrario, les prêts à bouger ont une forte probabilité de pratiquer ce type de mobilité, bien que l'absence du domicile, en lien avec des voyages fréquents, appelle de très bonnes compétences pour la gestion des plannings et de l'espace, pour communiquer dans diverses langues, etc. Les plus enclins à pratiquer ce genre de mobilité sont les non-réversibles (2.8 fois plus). En effet, ces derniers, s'ils ne sont que peu disposés à la bi-résidence, ne sont pas nécessairement opposés aux voyages fréquents, l'autre aspect de l'absence du domicile. Ils disposent surtout des accès et des compétences nécessaires à réaliser ce genre de mobilité dans de bonnes conditions.

À la lumière de ces analyses, il apparaît que les liens entre le potentiel et son activation (c.-à-d. entre la motilité et la grande mobilité) sont complexes. Les individus les mieux dotés en compétences et en accès paraissent ainsi plus libres d'adopter le comportement qu'ils souhaitent en matière de grande mobilité, et tout particulièrement de ne pas activer leurs compétences en la matière et de les garder à l'état de potentiel. Par ailleurs, la combinaison entre accès, compétences et disposition qu'offre la motilité révèle un grand pouvoir explicatif. En effet, les variables d'ordre sociodémographique utilisées (mis à part le genre et la composition du ménage) mettent en lumière des effets plus limités sur la grande mobilité des individus. Finalement, les plus motiles ne sont pas les plus mobiles, et les moins motiles ne sont pas les moins mobiles, ce qui nous amène à invalider l'hypothèse 3 . Par contre, la motilité permet de rendre compte du degré de choix des personnes en matière de grande mobilité. Les plus motiles sont effectivement ceux qui sont le plus en mesure de choisir entre être mobile et ne pas l'être ; les moins motiles sont les plus vulnérables face à l'injonction à la mobilité que peut engendrer le besoin de travailler.

\section{Conclusion}

Nous avons souhaité tester à travers cet article la pertinence du concept de motilité pour rendre compte de la variabilité des aptitudes à se mouvoir des personnes vis-à-vis de la grande mobilité.

La complexification des parcours familiaux et des carrières professionnelles incitent à adopter des pratiques de grande mobilité. Les comprendre nécessite d'analyser finement la manière dont les personnes et les ménages articulent spatialement vie privée et vie professionnelle par l'intermédiaire de leur motilité, dans un contexte où l'amélioration de l'offre de transport permet des déplacements plus rapides, plus lointains et plus fréquents. Les analyses réalisées débouchent sur deux résultats essentiels.
Les investigations ont tout d'abord montré une grande variabilité des types de motilité : certaines personnes en sont faiblement dotées alors que d'autres possèdent des aptitudes aux déplacements très étendues. Mais surtout, des rapports à l'espace très différents sont apparus. Certaines personnes sont peu ancrées à leur territoire de résidence, alors que d'autres y sont très attachés. Au-delà et parmi les plus ancrés, certains ont une grande capacité au déracinement et à l'enracinement dans un autre contexte, alors que d'autres sont fondamentalement sédentaires, même s'ils ont une grande aptitude à la pendularité. Ainsi, la motilité permet de distinguer des groupes de manière très fine et les individus qui les composent sont plus ou moins bien préparés à l'injonction à la flexibilité et à la grande mobilité qu'elle implique de plus en plus fréquemment.

Les travaux réalisés montrent deuxièmement que la motilité des personnes s'est révélée assez évolutive. Accéder à un emploi nécessite parfois de repenser les projets de mobilité vers une plus grande ouverture à la grande mobilité, même lorsqu'on ne dispose pas de toutes les compétences nécessaires. Le lien entre motilité et mobilité effective se révèle ainsi complexe. Si avoir de meilleurs accès, de meilleures compétences et de meilleurs projets ne se décline pas nécessairement par une plus grande pratique de la grande mobilité, les plus motiles se révèlent plus aptes à s'adapter aux exigences parfois difficiles à concilier des vies professionnelles et privées.

Mais au-delà de ces résultats, nous aimerions insister sur une limite du travail réalisé. La mesure de la motilité appliquée dans cet article, bien que fidèle à la description qui en est donnée dans la littérature, est perfectible à plusieurs niveaux. Les compétences considérées ne reflètent pas totalement le panel des savoirs nécessaires à la grande mobilité et à son bon déroulement, et les projets de mobilité, qui composent la troisième sphère de la typologie de la motilité, ont été approchés par le seul angle des dispositions déclarées par les personnes aux grandes mobilités. Sans remettre en cause la légitimité et la pertinence de nos analyses, ce travail mérite d'être poursuivi dans la perspective d'une approche plus complète et systématique de la motilité. Ainsi, un travail qualitatif mériterait d'être mené pour mettre en évidence les compétences nécessaires aux pratiques de grande mobilité, de même qu'un travail de réflexion sur la manière d'approcher les projets de mobilité serait nécessaire.

Malgré cette limite, nos résultats invitent à approfondir les analyses portant sur les aptitudes des personnes en matière de mobilité, car ils permettent de toucher des enjeux économiques et sociaux essentiels pour nos sociétés.

Les enjeux économiques renvoient en premier lieu aux questions de mobilité de la main-d'œuvre, généralement abordées sur la base des relocalisations et des migrations. Cependant, le développement des infrastructures de 
transport rapides et des télécommunications a favorisé l'émergence de formes hybrides de mobilité qui permettent d'occuper un emploi parfois très éloigné de son lieu de résidence, sans déménager. Notre travail montre ainsi à quel point les personnes ne sont pas dans des situations équivalentes face à ces formes de grande mobilité. Les enjeux sociaux en découlent directement. Du potentiel de grande mobilité à son activation, le degré de liberté des choix de vie se révèle très variable et les conséquences sur la vie privée et familiale (mais aussi la vie professionnelle sans doute) peuvent être importantes... Faut-il, alors, défendre unilatéralement un droit à la grande mobilité ?

\section{Remerciements}

Les données et analyses dont sont issus les travaux présentés dans cet article ont été financées par le FP7 de l'Union européenne et par le Forum Vies Mobiles - Institut SNCF de l'écomobilité. Les auteurs tiennent par ailleurs à remercier le Forum pour son soutien et tout particulièrement Christophe Gay et Sylvie Landriève pour leur implication dans le projet et leurs commentaires avisés.

\section{Bibliographie}

1. Schneider N, Meil G. (eds.) (2008) Mobile living across Europe I, Relevance and Diversity of Job-Related Spatial Mobility in Six European Countries. Barbara Budrich, Leverkusen Opladen.

2. Schneider N, Collet B (eds.) (2010) Mobile Living across Europe II. Causes and consequences of job-related spatial mobility in cross-national comparison. Barbara Budrich, Leverkusen Opladen.

3. Vogt W, Lenz M, Kalter F, et al. (2001) Die Bedeutung des täglichen Fernpendelns für den sekundär induzierten Verkehr. Berichte der Bundesanstalt für Straßenwesen, Verkehrstechnik. 88, Bergisch Gladbach.

4. Lück D, Rüppenthal S (2010) Mobile living: spread, appearances and characteristics. In: Schneider N., Collet B. (eds.), Mobile Living across Europe II. Causes and consequences of job-related spatial mobility in cross-national comparison. Barbara Budrich, Leverkusen Opladen.

5. Urry J (2005) Sociologie des mobilités : une nouvelle frontière pour la sociologie? Armand Colin, Paris.

6. Rosa H (2010) Accélération : Une critique sociale du temps. La Découverte, Paris.

7. Mongin O (2011) Questions de mobilité (connexions, vitesses, accès et espaces publics). In: Gay C., Kaufmann V., Landriève S., Vincent-Geslin S. (eds.), Mobile, Immobile, Choices and rights for 2030. Éditions de l'aube, La Tour d'Aigues : 86-96.

8. Bacqué M-H, Fol S (2007) L'inégalité face à la mobilité : du constat à l'injonction. Revue Suisse de Sociologie 33(1) : 89-104.

9. Kaufmann V (2008) Les paradoxes de la mobilité: bouger, $s$ 'enraciner. Presses polytechniques universitaires romandes, Lausanne.

10. van Houtum H, van der Velde M (2004) The power of cross-border labour and market immobility. Tijdschrift voor Economische en Sociale Geografi 95(1): 100-107.
11. van Wee B, Rietveld P, Meurs H (2006) Is average daily travel time expenditure constant? In search of explanations for an increase in average travel time. Journal of Transport Geography 14(2): 109-122.

12. Lyons G, Chatterjee K (2008) A Human Perspective on the Daily Commute: Costs, Benefits and Trade-offs. Transport Reviews 28(2): 181-198.

13. Sandow E (2011) On the road. Social aspects of commuting long distances to work. Umea University, Umea. PhD Thesis.

14. Vincent-Geslin S, Joly I (2012) Raisons et pratiques de la pendularité intensive. Le temps de trajet, entre temps subi et temps choisi. Cahiers scientifiques des transports 61: 159-189.

15. Ascher F (2004) Les sens du mouvement : modernités et mobilités. In: Allemand S., Ascher F., Lévy J. (eds.), Les sens du mouvement. Modernité et mobilités dans les sociétés urbaines contemporaines. Belin, Paris : 21-34.

16. Authier J-Y, Bonvalet C, Levy J-P (eds.) (2010): Élire domicile : la construction sociale des choix résidentiels. Presses universitaires de Lyon, Lyon.

17. Pochet P, Routhier J-L (2002) Mobilité résidentielle et allongement des distances domicile-travail dans la région urbaine de Lyon. In: Lévy J.-P., Dureau F. (eds.), L'accès à la Ville : Les mobilités spatiales en questions. L'Harmattan, Paris : 167-181.

18. Fischer PA, Malmberg G (2001) Settled People Don't Move: On Life Course and (Im-) Mobility in Sweden. International Journal of Population Geography 7(5): 357-371.

19. Vincent-Geslin S, Kaufmann V (2012) Mobilités sans racine. Plus loin, plus vite... Plus mobiles? Éditions Descartes \& Cie, Paris.

20. Thomas M-P (2013) Urbanisme et modes de vie. Enquête sur les choix résidentiels des familles en Suisse. Éditions Alphil-Pressses universitaires suisses, Neuchâtel.

21. Gillio N, Ravalet E (2012) Comprendre l'économie des territoires. Éditions du Certu, Lyon.

22. Ravalet E (2009) Ségrégation urbaine et mobilité quotidienne, une perspective internationale, Études de cas à Niamey, Puebla, Lyon et Montréal. INRS-UCS, Montréal, Université Lyon 2. Thèse de doctorat.

23. Wiel M (1999) La transition urbaine ou le passage de la ville pédestre à la ville motorisée. Mardaga, Sprimont.

24. Ley D (1996) The New Middle-Class and the Remaking ot the Central City. Oxford University Press, Oxford.

25. Florida R (2004) The rise of the creative class: and how it's transforming work, leisure, community and everyday life. Basic Books, New York.

26. Pattaroni L, Kaufmann V, Thomas M-P (2012) The Dynamics of Multifaceted Gentrification: A Comparative Analysis of the Trajectories of Six Neighbourhoods in the Île-de-France Region. International Journal of Urban and Regional Research 36(6): $1223-1241$.

27. Dieleman FM (2001) Modelling residential mobility; a review of recent trends in research. Journal of Housing and the Built Environment 16: 249-265.

28. Wenglenski S (2006) Regards sur la mobilité au travail des classes populaires. Une exploration du cas parisien. Cahiers scientifiques du transport 49 : 103-127.

29. Ahn N, De La Rica S, Ugidos A (1999) Willingness to Move for Work and Unemployment Duration in Spain. Economica 66(263): $335-357$.

30. Vignal C (2005) Injonctions à la mobilité, arbitrages résidentiels et délocalisation de l'emploi. Cahiers internationaux de sociologie 1(118) : 101-117.

31. Limmer R (2004) Job mobility and living arrangements In: Bonss W., Kesselring S., Vogl G. (eds.), Mobility and the cosmopolitan perspective. A workshop at the Munich Reflexive Modernization Research Centre, Munich: 89-94. 
32. Eliasson K, Lindgren U, Westerlund O (2003) Geographical Labour Mobility: Migration or Commuting? Regional Studies 37(8): 827-837.

33. Green AE, Hogarth T, Shackleton RE (1999) Longer distance commuting as a substitute for migration in Britain: a review of trends, issues and implications. International Journal of Population Geography 5(1): 49-67.

34. Scheiner J (2006) Housing mobility and travel behaviour: A process-oriented approach to spatial mobility: Evidence from a new research field in Germany. Journal of Transport Geography 14(4): 287-298.

35. van Ham M (2001) Workplace Mobility and Occupational Achievement. International Journal of Population Geography 7: 295-306.

36. Schneider NF, Limmer R, Ruckdeschel K (2002) Mobil, flexible, gebunden. Familie und Beruf in der mobilen Gesellschaft. Campus, Frankfurt am Main.

37. Hardill I, Green A (2003) Remote working — altering the spatial contours of work and home in the new economy. New Technology, Work and Employment 18(3): 212-222.

38. Bassand M, Brulhardt M-C (1980) Mobilité Spatiale. Georgi, StSaphorin.

39. Jansson B (1999) Migrate or Commute. Strategic household choices in Sweden during the 1990s. Association of American Geographers, 95th Annual AAG-99 Meeting. Honolulu.

40. McKenzie RD (1927) Spatial distance and community organization pattern. Social forces 5(4): 623-627.

41. Kaufmann V (2002) Re-Thinking Mobility: Contemporary Sociology. Ashgate Publishing Company, Farnham.
42. Boltanski L, Chiapello È (1999) Le nouvel esprit du capitalism. Gallimard, Paris.

43. Bourdin A (2005) Les mobilités et le programme de la sociologie. Cahiers internationaux de sociologie 118(1) : 5-21.

44. Kaufmann V, Bergman MM, Joye D (2004) Motility: Mobility as a capital. International Journal of Urban and Regional Research 28(4): 745-756.

45. Lévy J (1994) L'espace légitime. Sur la dimension géographique de la fonction politique. Presses de la fondation nationale des sciences politiques, Paris.

46. Lévy J (2003) Capital spatial. In: Lévy J., Lussault M. (eds.), Dictionnaire de la géographie et de l'espace des sociétés. Belin, Paris.

47. Urry J (2007) Social Inequalities in Network Capital. Swiss Journal of Sociology 33(1): 9-26.

48. Kaufmann V, Viry G, Widmer E (2010) Motility. In: Schneider N. F., Collet B. (eds.), Mobile Living Across Europe II, Causes and Consequences of Job-Related Spatial Mobility in Cross-National Comparison. Barbara Budrich Publishers, Opladen \& Farmington Hills : 95-111.

49. Piron M, Dureau F, Mullon C (2004): Utilisation de typologies multidates pour l'analyse des transformations socio-spatiales de Bogota (Colombie). Cybergeo : European Journal of Geography (En ligne). URL: http://cybergeo.revues.org/3742, $10 / 02 / 2014$

50. Fol S (2009) La mobilité des pauvres. Belin, Paris.

51. Commission Européenne (2012) Les Européens et leurs langues. Eurobaromètre spécial 386. Brussells. 